\title{
Structural probabilistic assessment of Offshore Wind Turbine operation fatigue based on Kriging interpolation
}

\author{
Rui Teixeira, Alan O’Connor \& Maria Nogal \\ Trinity College Dublin, Dublin, Ireland \\ James Nichols \& Mark Spring \\ Lloyd's Register, London, United Kingdom
}

\begin{abstract}
The probabilistic analysis of Offshore Wind Turbines (OWT) is not a new practice. The standards for designing OWT (IEC 61400 class) emphasizes that assessing uncertainty is of major importance inside the design chain. Still, major challenges related to the uncertainty and the probabilistic assessment pose to the sector and its development. The analysis of operational loads is one them.

The problem of analyzing extreme responses or cumulated damage in operation during the design phase is significantly related to its high computational cost. As we progressively add complexity to the system to account for its uncertainties, the computational effort increases and a perceptive design becomes a heavy task. If an optimization process is then sought, the designing effort grows even further. In the particular case of fatigue analysis, it is frequent to not be able to cover a full lifetime of simulations due to computational cost restrictions.

The mentioned difficulties fomented the utilization of surrogate models in the reliability analysis of OWT. From these surrogate approximations the ones based on Kriging models gained a special emphasis recently for structural reliability. It was shown that, for several applications, these models can be efficient and accurate to approximate the response of the system or the limit state surfaces.

The presented paper tackles some of the issues related to their applicability to OWT, in a case specific scenario of the tower component subjected to operational fatigue loads. A methodology to assess the reliability of the tower component to fatigue damage is presented. This methodology combines a Kriging model with the theory of extreme values. A one-dimensional Kriging case using the state of art NREL's monopile turbine is presented.The reliability of the OWT tower is calculated for 20 years.

The results show that the usage of a Kriging model to calculate the long term damage variation shows a high potential to assess the reliability of OWT towers to fatigue failure.
\end{abstract}

\section{INTRODUCTION}

The Offshore Wind Turbine (OWT) sector has been growing steadily in parallel with the current needs and demands for clean energy. Still, the cost of OWT energy is still above other energy conversion sources. Despite being a very highly technical sector, improvement of the design techniques remains a need to unlock new breakthroughs that will enable it to become progressively more competitive.

At the same time, probabilistic design is further demanded by the sector as a strategy to enable comprehensive designing processes that generate robust systems.
One of the areas of particular concern for OWT, as designed for long life-time, is the fatigue design. In the case of the OWT towers, fatigue is seen as a major driver of failure.

Certifying and designing a wind turbine to ensure that it is not going to fail through structural fatigue may involve a very onerous design process. The calculation of fatigue on a wind turbine, as a cumulative event, means that the designer needs to simulate and replicate the damage suffered by the wind turbine during its design life time. This usually means thousands of computational simulations, and consequently, a huge computational effort that hinders the design and even more the optimization process on a practical basis. 
To simplify the process a simplified analysis that prioritizes the most critical enviromental states (or the ones expected to originate more damage) is frequently applied. Running all the simulations that represent the full lifetime of a OWT is not a common practice. Even with several simplifications and assumptions the fatigue remains a very highly resource demanding task.

Due to its non-linear character and to some of the assumptions and simplifications considered, uncertainty propagates through the design chain interfering with the awareness of the real robustness of the system.

Under this context, the following paper presents a methodology for the calculation of offshore wind turbine towers fatigue based on a surface model. The usage of a surface model, in the case a Kriging model, reduces significantly the amount of computational effort needed to address the problem of fatigue. A methodology for calculation of long-term fatigue on OWT towers is presented and discussed. It is important to highlight that the methodology presented here for OWT towers can be applied to other structural component.

To successfully introduce the proposed methodology, Section 2 presents the main theoretical aspects of the reliability assessment based on Kriging models and some of the most relevant works up to date. Section 3 presents the methodology applied and the model used for analysis. Section 4 shows the main results of the analysis and discusses them allowing for the main conclusions to be drawn in the following chapter.

\section{FATIGUE RELIABILITY OF OWT}

The design of OWT stands to two major standards, the IEC61400-1 (IEC, 2005) and IEC61400-3 (IEC, 2009). Other standards, guidelines and recomendations can be found that comply with the previously mentioned and that were created to help the designer during design and certification processes, e.g. (DNV, 2014).

The current design practices implemented in the industry in regard of fatigue assessment have more of deterministic than probabilistic. To assess fatigue failure, commonly a set of simulations are run and the damage is assessed for the design lifetime with some subjacent considerations that minimize the computational effort. Assessment of the uncertainties and the variations introduced by in the process is limited.

For fatigue analysis the assumption of linearly cumulative damage represented by the Miner's rule with partial safety factors is endorsed. To extract the damage generated by the external loads a rainflow counting methodology is recommended (DNV, 2014).

Even introducing several simplifications, the analysis of fatigue of an OWT accordingly to the certification requirements still represents a highly resource in- tensive task that can involve several hundreds or thousands of simulations of heavy and complex models. It is of great interest then to introduce and validate methodologies that can significantly reduce this effort and increase the precision of the design.

In Kallehave et al. (2015) it is mentioned that depending on the actual structural detail, there may be a further potential to extend the lifetime by up to $40 \%$, or equivalently reduce the mass of fatigue driven parts of the pile in future designs.

Several challenges need to be tackled though, it is shown in Sutherland and Veers (1995), through performing experimental tests on a 34 meters blade, that the damage calculated in 10 minutes can vary substantially.

Therefore, even considering the significant potential to improve on fatigue assessment, any further methodology needs to be meticulous with inherent uncertainties.

One of the methodologies that has been gaining particular notoriety to account for the uncertainties in the response of the physical systems is the use of surrogate models, in particular Kriging surrogate models. The following chapter presents the theory subjacent to these models and some of the most relevant works on structural reliability and fatigue analysis where they were applied.

\subsection{Surrogate Kriging Models}

A surrogate model or metamodel is a generic description that includes different types of models. These can be usually described as a model of a model. They can be also understood as a mathematical relation or an algorithm that relates the inputs and the outputs of a certain system or process. These approximation models are many times also described as, response surface methods, in the sense that they mimic the behaviour of a simulation model as close as possible with a surface, having as a direct benefit the reduction of the computational cost of evaluating that model.

The most popular surrogate models are polynomial response surfaces, Kriging models, support vector machines, space mapping, and artificial neural networks. In the present work attention is given to the Kriging models which the application in structural reliability is relatively recent and is an active area of research lately. The Kriging surface models present the big advantage of considering the uncertainty in the approximation of a true response of a model, making it therefore interesting for reliability engineering.

The principle behind the Kriging models is to approximate the true state function $g(x)$ that depends on a vector $x=\left[X_{1}, X_{2}, \ldots, X_{n}\right]$ of $n$ random variables with an approximate mathematical model $G(x)$. 
The Kriging models are exact interpolators based on the idealization of the numerical model response as the realization of a Gaussian stochastic process (Echard et al., 2011). Therefore, with a Kriging model the true response function $g(x)$ can be written as follows:

$G(x)=f(\beta ; x)+z(x)$

$f(\beta ; x)=\beta_{1} f_{1}(x)+\ldots+\beta_{p} f_{p}(x)$

where $f(\beta ; x)$ is a deterministic component determined by a regression model defined by $p$ basis functions $f_{p}(x)$ and $p$ regression coefficients $\beta$; being the number of $p$ coefficients needed to estimate function of the approximation intended in the regression (order considered). On the other hand, $z(x)$ is a Gaussian stochastic process with zero mean and covariance between two points in the space:

$\operatorname{cov}\left(z\left(x_{i}\right), z\left(x_{j}\right)\right)=\sigma_{z}^{2} \Omega\left(\theta ; x_{i}, x_{j}\right)$,

with $i, j=1,2,3, \ldots, m$

here, $\sigma_{z}^{2}$ is the constant process variance and $\Omega$ a correlation function, which represents the correlation between two arbitrary points $x_{i}$ and $x_{j}$ in the space. The process of fitting a Kriging metamodel demands a sample of $m$ support points or observations that is commonly designated as Design of Experiments (DoE); $D o E=\left[x_{k}, y_{k}==g\left(x_{k}\right)\right]$ with $k=1,2,3, \ldots m$. In this sample of support points the Kriging prediction is the exact value realisation at each $x_{k}$. The covariance function interpolates then the errors between the regression model predictions and the true limit state realisations. In the framework of structural analysis an exponential Gaussian correlation function has been widely applied for structural reliability analysis.

$\Omega\left(\theta ; x_{i}, x_{j}\right)=\exp \left(-\sum_{i=1}^{n} \theta_{i}\left(x_{i}-x_{j}\right)^{2}\right)$,

where $n$ is the dimension of the vector space, also it is the number of random variables. The correlation function depends on the realisations $x_{i}$ and $x_{j}$ and on the correlation parameters $\theta_{i}$.

It can be seen that the Kriging model depends on 3 main parameters, the regression coefficients $\beta$, the variance $\sigma_{z}^{2}$ and the correlation parameters $\theta$. For a given sample of support points the problem can then be solved through a generalised least squares formulation, where the estimator for $\hat{\beta}$ and $\hat{\sigma}_{z}^{2}$ are given by the following equations.

$$
\begin{aligned}
& \hat{\beta}=\left(F^{T} \Omega^{-1} F\right)^{-1} F^{T} \Omega^{-1} y_{1: m} \\
& \hat{\sigma}_{z}^{2}=\frac{1}{m}\left(y_{1: m}-F \hat{\beta}\right)^{T} \Omega^{-1}\left(y_{1: m}-F \hat{\beta}\right)
\end{aligned}
$$

$F$ is the regression matrix in which the lines are the basis functions $f\left(\beta, x_{k}\right)$ evaluated at the $m$ support points and $y_{1: m}$ is the vector of true realisations of the function as previously defined at $m$ points. The matrix $\Omega$ defines the correlation between each pair of support points according to the correlation function considered before:

$\Omega=\left[\begin{array}{ccc}\Omega\left(x_{1}, x_{1}\right) & \ldots & \Omega\left(x_{1}, x_{m}\right) \\ \vdots & \ddots & \vdots \\ \Omega\left(x_{m}, x_{1}\right) & \ldots & \Omega\left(x_{m}, x_{m}\right)\end{array}\right]$

This matrix, as can be seen in Equation 4, depends on the parameter $\theta$ and therefore its calculation is needed before assessing the value of both $\hat{\beta}$ and $\hat{\sigma}_{z}^{2}$. The correlation function is then defined by using maximum likelihood estimation:

$\hat{\theta}=\operatorname{argmin} L(\theta)$

with,

$L(\theta)=(\operatorname{det} \Omega)^{\frac{1}{m}} \hat{\sigma}(\theta)_{z}^{2}$

being the $L(\theta)$ the likelihood estimator to be minimised. Knowing all the estimators for the Kriging model, a prediction of $g(u)$ in a certain point $u$ in the space is given by the Kriging expected value $\mu_{G}$ and variance $\sigma_{G}^{2}$ :

$\mu_{G}(u)=f(u)^{T} \hat{\beta}+r(u)^{T} \Omega^{-1}(y-F \hat{\beta})$

$\sigma_{G}^{2}=\sigma_{z}^{2}\left(1+U(u)^{T}\left(F^{T} \Omega^{-1} F\right)^{-1} U(u)-r(u)^{T} \Omega^{-1} r(u)\right)$

$U(u)=F^{T} \Omega^{-1} r(u)-1$

$r(u)=\left[\Omega(u, x 1), \ldots, \Omega\left(u, x_{m}\right)\right]$ is the correlation vector between one point and all the other known points. Not forgetting that $f(u)$, like defined before, is the trend of the model, the deterministic part of it.

A zero order polynomial is of common application, meaning that Equation 2 reduces to $f(\beta, x)=\beta_{1}$. This means that the matrix $F$ for this case takes the form of a vector with components $f_{k}\left(x_{k}\right)=1$ for $m$ components. It is assumed that a constant term is sufficient for generating precise Kriging interpolation models for reliability. In Gaspar et al. (2014) it is shown that higher order polynomials may not contribute to improved accuracy in the case particular structural of reliability engineering problems.

It is important to emphasize that in a Kriging model a function is approximated in a way that it is exactly predicted in a point $u$ that belongs to the space of experiments or sample of support points, meaning that $\mu_{G}(u)$ is exactly $G(u)$ and its Kriging variance is null. 
The Kriging models or Gaussian process models are of interest for the topic of reliability analysis due to their interpolation capacity, the flexibility to approximate arbitrary functions with a high level of accuracy and the capability of accounting for a local uncertainty measure.

\subsection{Kriging model in reliability analysis of OWT}

Examples of the application of the Kriging models for reliability problems can be found in (Bichon et al., 2008; Echard et al., 2011; Zhang et al., 2015; Gaspar et al., 2014). In the particular case of the reliability analysis of offshore wind turbines, the application of Kriging surface to calculate the reliability of the system is relatively new.

The application of Kriging models for OWT reliability analysis was first highlighted in Yang et al. (2015), where a reliability based probabilistic optimization of a Tripod foundation was developed. The variable of optimization considered was the weight of the structure which was then subjected to specific constraints, and the reliability checked through MonteCarlo simulations. The advantage of using a metamodel to reproduce the behaviour of the system is well shown, as a more comprehensive design is achieved through the process with a reduced amount of computational time.

Following, in Morató et al. (2016) the response of an OWT is modeled using a Kriging model. Again, approximating the response of the model with a Kriging surface is motivated by the reduction of the computational time needed to analyse the reliability of an OWT. Two limit state (LS) functions are considered in the analysis. As in Yang et al. (2015) Latin Hypercube sampling is used to set the design of experiments.

While these models steadily gained interest for analysing OWTs, their usage in the wind energy industry dates from before. In Maki et al. (2012) the wind turbine response is approximated with a Kriging model and therefore applied to replace the computational expensive coupled dynamic code. This enables a multi-level optimization approach of the blades of a $1 \mathrm{MW}$ wind turbine.

When analyzing the fatigue life of components, the use of Kriging is not widely common. On the other hand, the potential shown for its application is high. Echard et al. (2013) reduced the computational time of a fatigue reliability analysis from more than 100 days to approximately 9 hours by using a surrogate model and adaptive sampling methodologies. Showing therefore the huge benefits in reduction of the computational cost from the application of a Kriging interpolation scheme without compromising accuracy in the results. In Yang and Wang (2012) a probabilistic structural fatigue resistance optimization of a bending stiffener is presented where they compare three metamodels, among which there is a Kringing interpolation surface, to reduce computational time and enable the optimization to be practicable.

In the particular case of fatigue analysis of OWT no work was found that combines the Kriging models and the fatigue analysis. A methodology for the fatigue analysis of OWT will be presented in the following chapter and the uncertainty resulting from its application addressed.

\section{OWT FATIGUE ANALYSIS MODELLING}

In the present study NREL's 5MW baseline OWT is applied due to its state of the art character, (Jonkman et al., 2009). Uncertainty quantification toolbox, UQLab Marelli and Sudret (2014), was used to build the Kriging model. The structural analysis is, as mentioned, focused on the tower and the loads (foreaft moment) are calculated in 9 points across its height. To present the method used, the analysis here focuses on the point located in the connection with the interface piece which is expected to be the more critical one. In further analysis of the uncertainty of the method, all the points will be considered to infer on the influence of change of cross section.

It is usually assumed in the bibliography that under constant or random loading the fatigue life follows a normal or log-normal distribution (Wu et al., 1997; Wirsching and Chen, 1988). This is an important assumption for the methodology proposed as this way, it is correct to assume that a Kriging surface will reproduce long-term fatigue results adequately.

To assess the fatigue life of the structure a new methodology that is aligned with the current practices used in the sector for the fatigue analysis of OWT is introduced. Ten minutes simulations are used to determine the short term levels of fatigue damage. To calculate the short term damage the current practice used in the sector is applied. Rainflow counting method is therefore used to assess the number of cycles associated to each stress interval (Niesłony, 2009). The long term fatigue calculations assume linear cumulative damage accordingly to the Miner's rule, Equation 13 ,

$D_{C}=\sum_{i=1}^{I} \frac{n_{i}}{N_{i}}$

where $n_{i}$ are the number of cycles experienced at a certain stress bin $i$ during the lifetime and $N_{i}$ is the theoretical maximum according to the $\mathrm{S}-\mathrm{N}$ curve, the damage level can then be determined individually for each of the life time ten minutes cycles experienced 
by the turbine. The fact that the damage can be linearly summed allows the definition of a surface that represents the damage experienced by the OWT. This surface can then be approximated by a Kriging interpolation scheme, reducing as a consequence the number of simulations needed to have a full assessment of the OWT $n_{i}$ cycles for each stress bin during the expected life time.

It is important to highlight that in the current industry applications the designer may choose the environmental states considered more important, or the ones expected to contribute with more structural damage, as running all the life-time simulations may be impracticable. The usage of lumped scatter diagrams is piratical representation of this, (Fischer et al., 2010). As a consequence, the simulation time can be quite reduced, but the approach originates less awareness of the real behaviour of the OWT and may overestimate or underestimate the real damage levels.

To fully assess the fatigue life of the OWT tower after calculating the damage surface two proposals are presented: the usage of Monte-Carlo simulations from the originated Kriging interpolation, or the integration of the different levels of damage through the expected joint distribution of environmental variables during the life-time of the turbine.

Since the focus here is to validate the model and assess some of its errors, no special methodology is used to generate the DoE. In the future special attention should be paid to the way the design of experiments is picked in order to increase the efficiency of applying the methodology. The damage levels are assessed in this phase based on the main environmental variable: wind velocity $\left(v_{\text {wind }}\right)$. Either way, Kriging models are shown to work well when the space of variables increases (Gaspar et al., 2014).

\section{DAMAGE SURFACE APPROXIMATION WITH KRIGING}

To analyse the results of applying the described methodology a Kriging metamodel was built with the wind velocity $\left(v_{\text {wind }}\right)$ as the main variable of the DoE. The wind is expected to be the main contributor for the damage in the tower. Other variables, e.g. the wave conditions, direction of the wind, among other, are expected also to contribute to damage of the OWT. To generate the response surface first a single seeded simulation was compared with results from six seeded wind speeds.

Important to notice that every simulation of the coupled dynamic code takes an average time of 20 to 25 minutes, making the analysis very resource intensive in case a full life-time simulation is pursuit. In the industry the fatigue calculation for OWT is addressed by se- lecting the simulations expected to contribute the most for the life-time. The end result is a single point assessment for twenty years were the result accounts for a very limited probabilistic assessment.

The results of the Kriging fitting for a one dimensional variable are shown in Figure 1. The Kriging Gaussian uncertainty approximates the short term damage variability and generates random realisations (simulated points) that replicate the fatigue uncertainty. An ordinary Kriging metamodel was considered in the model.

Despite being a state-of-art code, the calculations of the short-term loads and the short-term damage generated were validated with the results from the commercial software GH-Bladed. Even considering that it is unlikely to generate two equal time series, the order of the damage and the Markov matrices were compared for reference.

For the calculation of the fatigue a bi-linear S-N curve was adopted accordingly with the guidelines given in DNV (2014).
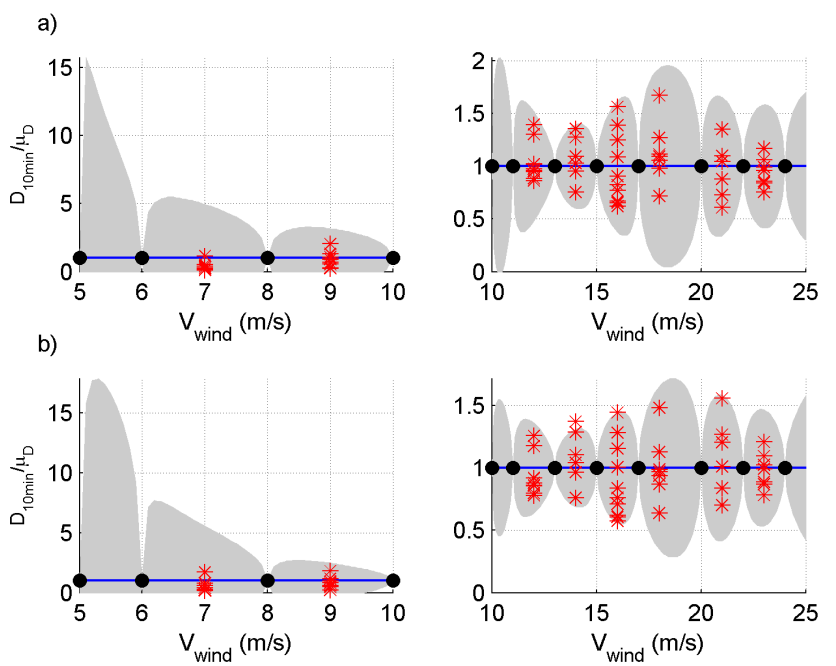

$95 \%$ Confidence Intervals

- DoE $\quad$ * Simulated points

Figure 1: a) Kriging model not considering local variability of the DoE. b) Kriging model considering local variability of the DoE.

It is possible to identify that not considering the short-term variability of the damage may introduce substantial errors in the Kriging prediction. With a higher number of seeds the Kriging model approximates the response in a more adequate way. It is possible to use a small number of points, but the variation in the surface is much bigger as the result of a one or two simulations can approximate the real mean value if exactly the right points are picked, which is highly unlikely.

For low wind speeds $(<10 \mathrm{~m} / \mathrm{s})$ the standard deviation seems to be highly over predicted by the meta- 
model. This may be related to the low damage generated during this regime of operation. The damage generated in 10 minutes for wind speeds below the rated power does not contribute significantly for the total damage in 20 years as the damage in 10 minutes can decrese up to 100 when the wind velocities decreases in comparison with the damage originated at rated power. Therefore, to estimate the long-term variability of the damage wind speeds below $9 \mathrm{~m} / \mathrm{s}$ was considered as having the constant mean value predicted by the metamodel. Further analysis of using the Kriging surface for fatigue estimation of OWT may address this issue, although, its contribution for the operation fatigue life is residual. The increase of the number of points in the DoE that covers the low wind speeds is very likely to minimize this variability however the computational cost associated with refining the DoE in the low wind speeds should be evaluated against the additional accuracy gained in the long term estimation of damage.

Even considering that the Kriging surface seems to catch some of the variability of the short-term damage as the number of seeds increases, in Figure 1 it is possible to identify that it can under-predict or over-predict this variability.

The convergence of the results was then checked with the increase of the number of seeded wind velocities, using additional simulations to cross check the model. Due to computational time restrictions the initial six seeds were considered. The convergence of the mean and standard deviation was then checked to infer on the error of the metamodel approximation, Figure 2.
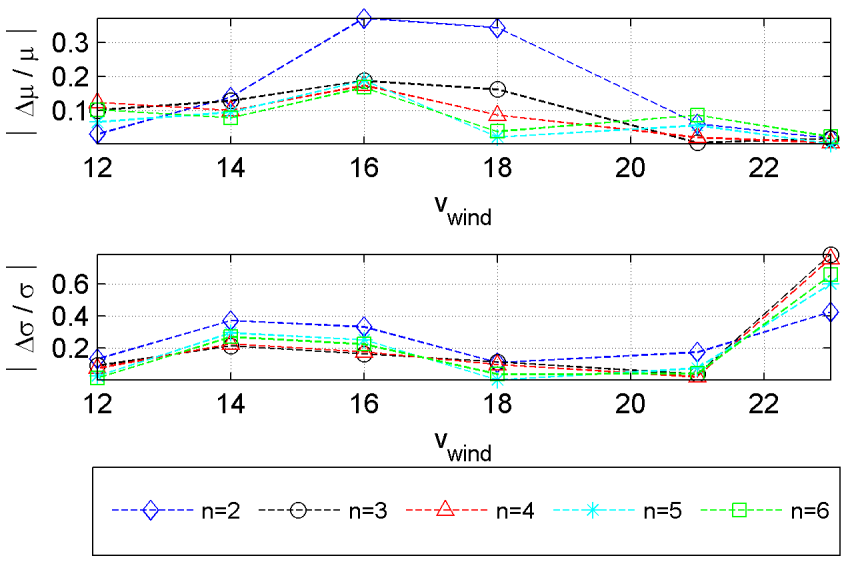

Figure 2: Convergence error of the mean and the standard deviation of the Kriging model.

The results show that the mean has an average error inferior to $10 \%$ while the standard deviation despite converging to values around $20 \%$ in the most important wind speeds, for high wind speeds it has a significant error (as can be seen in the confidence intervals of Figure 1), around $60 \%$. One of the potential factors that may be affecting the results at high $v_{\text {wind }}$ is the fact that the controller implemented in the NREL 5MW monopile turbine does not consider the shut-down of the turbine. Low wind speeds were not considered in this analysis of convergence due to the cited reasons and the difficulty in having proper estimations of their uncertainty.

It is important to notice that the present graphic indicates, using the example of when 3 points are applied to create the Kriging, that the accuracy of the model will depend more on the points picked for the DoE than on the number of points used. The mean is a measure that converges fast, while the predicted standard deviation is highly dependent on the picked points. Due to the normal distributed character of the damage one additional point may not mean better results until a certain threshold of number of points is achieved. The results show that a careful approach to the number of seeds simulated is needed in order to have the best trade-off in improvement of accuracy when comparing with the additional cost of generating new points.

Nevertheless, the presented convergence graphic is very interesting in the sense that allows for a establishing a correction of the Kriging estimations in certain points. With few additional simulations it is possible to have a measure of the uncertainty in the Kriging predictions and with a coupled analysis have more accurate results. Following works shall address how this measure of uncertainty can support the selection of the DoE.

One of the biggest challenges in the application of the Kriging surface for fatigue is making these two measures converge with the model results.

If too many points are used to generate the Kriging metamodel, it can get constrained to the design of experiments and lose the capability to correctly predict the variability of the damage generated in 10 minutes. On the other hand, if few or badly chosen DoE points are considered, the model will overestimate the variability of the damage generated in 10 minutes.

Further developments shall then assess the DoE by characterizing the fatigue variability with the variability of the loads generated by the wind. Comparison of the results obtained from a implemented Kriging approach should be also compared with the ones obtained by a stochastic Kriging approach.

Even considering that during the lifetime the results will converge to the mean, neglecting the variability of the DoE and predicting its damage exactly in the model, may introduce errors by not considering important variations around those points when assessing the long term variability of the damage. This can be identified in Figure 3.

To calculate the extreme response of the damage a 


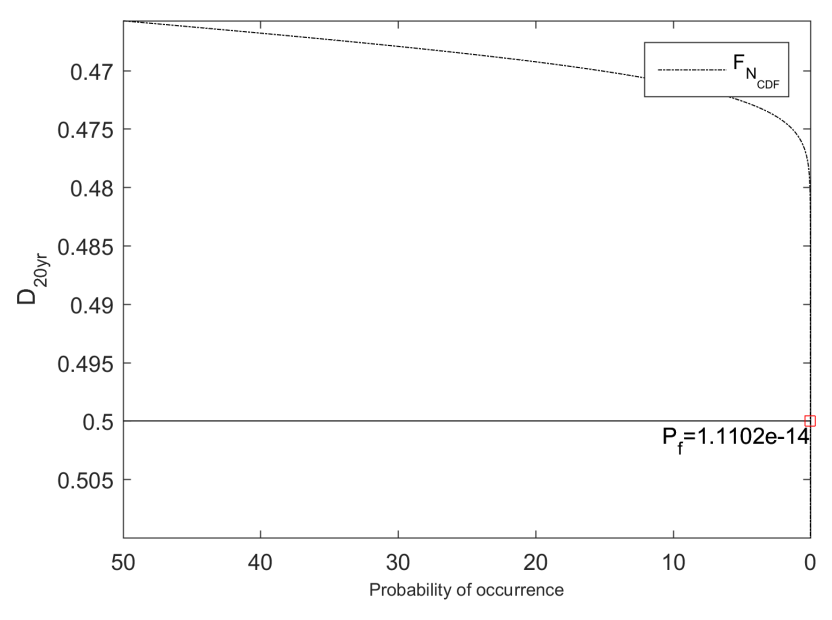

Figure 3: Extrapolated $P_{f}$ for the OWT tower fatigue

set of points was generated and then $P_{f}$ was extrapolated. The Kriging metamodel is applied to generate long term series in a very fast way when compared to what would be expected if all the simulations were run.

One hundred series of 20 years damage were generated from the Kriging surface. It is known that a linear combination of a set of Gaussian variables will produce a new Gaussian variable, so a normal distribution was used to fit the data. The long term damage distribution allows then the estimation of the probability of failure due to fatigue by calculating the probability of achieving the failure damage level.

The limit-state line of damage considered was the $\sum D=0.5$. The value of 0.5 appears in different documents that refer to the structural fatigue analysis due to the common overestimation of fatigue life by the assumption of linear summation of damage accordingly to the Palmgren-Miner rule, Equation 13, (Veldkamp, 2006). This design lifetime threshold can be found in some guidelines for OWT design by the recommendation of a design fatigue factor(DFF) for a lifetime without inspections, e.g. DNV (2014). It is possible to identify that $P_{f}$ is almost 0 for the considered structure in the framework presented. The analysed system's tower is expected to be highly reliable. As example, in Morató et al. (2016), couldn't compute its $P_{f}$ for the limit-state involving the extreme overturning moment of the tower. Nevertheless, several assumptions were taken in the current work in regard of the way the loading variables were analysed that may contribute to the calculated reliability (e.g. one single variable was used to create the Kriging model). In Veldkamp (2006) an extensive analysis of the uncertainty associated with the different design variables that rule the fatigue life of wind turbines is presented. It is then expected for a more extensive analysis of the different uncertainties in the design variables to increase the risk of failure. It is also important to highlight that the S-N curve has some inherent reliability concept that is associated with a probability of failure, which should be accounted and coupled in further computations of the shown probabilities.

\section{CONCLUSIONS}

An alternative approach to calculation of the fatigue reliability of OWT towers was presented in the present paper. This approach considers the utilization of a metamodel to approximate the fatigue damage generated in ten minutes. In the present case a Kriging model is applied due to its inherent capability to approximate the response field with a Gaussian process.

Using metamodels, in special the Kriging models, is not a new practice in the field of reliability. Although a robust approach is still needed for many specific case scenarios. For OWT towers the assessment of fatigue life in the design certification process usually disregards a reliability approach, being the survivability addressed as a deterministic process. This urged the need to develop methodologies to assess how robust is the design in the expected life time.

The Kriging models present as the main advantage the low computational cost. Additionally, they capture the uncertainty of the short-term damage. Their implementation is not intrusive in the source code, and the predictions for long term show the potential to be highly accurate.

Nonetheless, several challenges need to be tackled to improve the implementation of the presented methodology, being the four main ones:

- Address the uncertainty generated by considering the DoE as a deterministic prediction in the Kriging.

- Optimize the generation of DoE points to improve the accuracy in predicting the variability of the damage and having the least computational cost.

- Improve the Kriging accuracy for the variability in the low wind speed velocities.

- Increase the number of dimensions and analyse the accuracy and sensitivity of the results.

There are other more detailed challenge to be addressed as the possibility of missing out with this approach some narrow peaks of damage caused by resonance conditions. Surpassing these challenges may contribute to significant improvement in the ratio of effort and accuracy given by the model. The high short term fatigue damage variability has shown as being of major importance in future developments of the methodology. 
To conclude it is important to highlight that the approach presented significantly increases the designer awareness for the risks inherent to the fatigue life-time allowing a better planning of the life-cycle management of the equipment.

\section{ACKNOWLEDGEMENTS}

This project has received funding from the European Union Horizon 2020 research and innovation programme under the Marie Skłodowska-Curie grant agreement No. 642453.

\section{REFERENCES}

Bichon, B. J., M. S. Eldred, L. P. Swiler, S. Mahadevan, \& J. M. McFarland 2008 . Efficient global reliability analysis for nonlinear implicit performance functions. AIAA journal 46(10), 2459-2468.

DNV 2014 . Design of offshore wind turbine structures. Offshore Standard DNV-OS-J101, DNV GL AS.

Echard, B., N. Gayton, \& M. Lemaire 2011 . Akmcs: an active learning reliability method combining kriging and monte carlo simulation. Structural Safety 33(2), 145-154.

Echard, B., N. Gayton, M. Lemaire, \& N. Relun 2013 . A combined importance sampling and kriging reliability method for small failure probabilities with time-demanding numerical models. Reliability Engineering \& System Safety 111, 232-240.

Fischer, T., W. De Vries, \& B. Schmidt 2010 . Upwind design basis (wp4: offshore foundations and support structures).

Gaspar, B., A. Teixeira, \& C. G. Soares 2014 . Assessment of the efficiency of kriging surrogate models for structural reliability analysis. Probabilistic Engineering Mechanics 37, 24-34.

IEC 2005 . Wind turbines part 1: Design requirements. Technical Report 61400-1, International Electrotechnical Commission, Geneva, Switzerland.

IEC 2009 . Wind turbines part 3: Design requirements for offshore wind turbines. Technical Report 61400-3, International Electrotechnical Commission, Geneva, Switzerland.

Jonkman, J., S. Butterfield, W. Musial, \& G. Scott 2009 . Definition of a 5-mw reference wind turbine for offshore system development. National Renewable Energy Laboratory, Golden, CO, Technical Report No. NREL/TP-500-38060.

Kallehave, D., B. W. Byrne, C. L. Thilsted, \& K. K. Mikkelsen 2015 . Optimization of monopiles for offshore wind turbines. Phil. Trans. R. Soc. A 373(2035), 20140100.

Maki, K., R. Sbragio, \& N. Vlahopoulos 2012 . System design of a wind turbine using a multi-level optimization approach. Renewable Energy 43, 101110.

Marelli, S. \& B. Sudret 2014 . Uqlab: A framework for uncertainty in matlab. In Proceedings 2 nd Int. Conf. on Vulnerability, Risk Analysis and Management (ICVRAM), pp. 2554-2563.

Morató, A., S. Sriramula, \& N. Krishnan 2016 . Reliability analysis of offshore wind turbine support structures using kriging models. In Proc. ESREL Conference, Glasgow, September.

Niesłony, A. 2009 . Determination of fragments of multiaxial service loading strongly influencing the fatigue of machine components. Mechanical Systems and Signal Processing 23(8), 2712-2721.

Sutherland, H. J. \& P. S. Veers 1995 . Effects of cyclic stress distribution models on fatigue life predictions. Wind Energy 16, 83-90.

Veldkamp, H. F. 2006 . Chances in wind energy: a probalistic approach to wind turbine fatigue design. Ph. D. thesis, TU Delft, Delft University of Technology.

Wirsching, P. H. \& Y.-N. Chen 1988 . Considerations of probability-based fatigue design for marine structures. Marine Structures 1(1), 23-45.

Wu, W., H. Liou, \& H. Tse 1997 . Estimation of fatigue damage and fatigue life of components under random loading. International journal of pressure vessels and piping 72(3), 243-249.

Yang, H. \& A. Wang 2012 . Fatigue reliability based design optimization of bending stiffener. Journal of ship Research 56(2), 120-128.

Yang, H., Y. Zhu, Q. Lu, \& J. Zhang 2015 . Dynamic reliability based design optimization of the tripod sub-structure of offshore wind turbines. Renewable Energy 78, 16-25.

Zhang, L., Z. Lu, \& P. Wang 2015 . Efficient structural reliability analysis method based on advanced kriging model. Applied Mathematical Modelling 39(2), 781-793. 